\title{
Gamma-Ray Bursts as a Probe of the Very High Redshift Universe
}

\author{
Donald Q. Lamb and Daniel E. Reichart \\ Department of Astronomy $\&$ Astrophysics, University of Chicago, \\ 5640 South Ellis Avenue, Chicago, IL 60637
}

\begin{abstract}
We show that, if many GRBs are indeed produced by the collapse of massive stars, GRBs and their afterglows provide a powerful probe of the very high redshift $(z \gtrsim 5)$ universe.
\end{abstract}

\section{INTRODUCTION}

There is increasingly strong evidence that gamma-ray bursts (GRBs) are associated with star-forming galaxies $[1,2,3,4]$ and occur near or in the star-forming regions of these galaxies $[5,3,4,6,2]$. These associations provide indirect evidence that at least the long GRBs detected by BeppoSAX are a result of the collapse of massive stars. The discovery of what appear to be supernova components in the afterglows of GRBs $970228[7,8]$ and 980326 [9] provides direct evidence that at least some GRBs are related to the deaths of massive stars, as predicted by the widelydiscussed collapsar model of GRBs $[10,11,12,13,14,15]$. If GRBs are indeed related to the collapse of massive stars, one expects the GRB rate to be approximately proportional to the star-formation rate (SFR).

\section{GRBS AS A PROBE OF STAR FORMATION}

Observational estimates $[16,17,18,19]$ indicate that the SFR in the universe was about 15 times larger at a redshift $z \approx 1$ than it is today. The data at higher redshifts from the Hubble Deep Field (HDF) in the north suggests a peak in the SFR at $z \approx 1-2$ [19], but the actual situation is highly uncertain. However, theoretical calculations show that the birth rate of Pop III stars produces a peak in the SFR in the universe at redshifts $16 \lesssim z \lesssim 20$, while the birth rate of Pop II stars produces a much larger and broader peak at redshifts $2 \lesssim z \lesssim 10[20,21,22]$. Therefore one expects GRBs to occur out to at least $z \approx 10$ and possibly $z \approx 15-20$, redshifts that are far larger than those expected for the most distant quasars. Consequently 


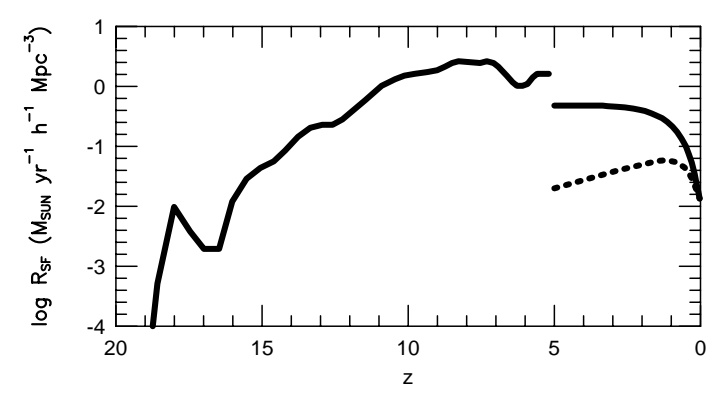

FIGURE 1. The cosmic SFR $R_{S F}$ as a function of redshift $z$. The solid curve at $z<5$ is the SFR derived by [23]; the solid curve at $z \geq 5$ is the SFR calculated by [21] (the dip in this curve at $z \approx 6$ is an artifact of their numerical simulation). The dotted curve is the SFR derived by [19].

GRBs may be a powerful probe of the star-formation history of the universe, and particularly of the SFR at VHRs.

In Figure 1, we have plotted the SFR versus redshift from a phenomenological fit [23] to the SFR derived from submillimeter, infrared, and UV data at redshifts $z<5$, and from a numerical simulation by [21] at redshifts $z \geq 5$. The simulations done by $[21]$ indicate that the SFR increases with increasing redshift until $z \approx 10$, at which point it levels off. The smaller peak in the SFR at $z \approx 18$ corresponds to the formation of Population III stars, brought on by cooling by molecular hydrogen. Since GRBs are detectable at these VHRs and their redshifts may be measurable from the absorption-line systems and the Ly $\alpha$ break in the afterglows [24], if the GRB rate is proportional to the SFR, then GRBs could provide unique information about the star-formation history of the VHR universe.

More easily but less informatively, one can examine the GRB peak photon flux distribution $N_{G R B}(P)$. To illustrate this, we have calculated the expected GRB peak flux distribution assuming (1) that the GRB rate is proportional to the $\mathrm{SFR}^{1}$, (2) that the SFR is that given in Figure 1, and (3) that the peak photon luminosity distribution $f\left(L_{P}\right)$ of the bursts is independent of $z$. There is a mis-match of about a factor of three between the $z<5$ and $z \geq 5$ regimes. However, estimates of the star formation rate are uncertain by at least this amount in both regimes. We have therefore chosen to match the two regimes smoothly to one another, in order to avoid creating a discontinuity in the GRB peak flux distribution that would be entirely an artifact of this mis-match.

For a peak luminosity function $f\left(L_{P}\right)$ and for $d L_{P} / d \nu \propto \nu^{-\alpha}$, the observed GRB peak flux distribution $N_{G R B}(P)$ is given by the following convolution integration:

$$
N_{G R B}(P)=\Delta T_{\text {obs }} \int_{0}^{\infty} R_{G R B}\left(P \mid L_{P}\right) f\left[L_{P}-4 \pi D^{2}(z)(1+z)^{\alpha} P\right] d L_{P},
$$

where $\Delta T_{o b s}$ is the length of time of observation, $D(z)$ is comoving distance,

$$
R_{G R B}\left(P \mid L_{P}\right) \propto \frac{R_{S F}(z)}{1+z} \frac{d V(z)}{d z}\left|\frac{d z\left(P \mid L_{P}\right)}{d P}\right|,
$$

1) This may underestimate the GRB rate at VHRs since it is generally thought that the initial mass function will be tilted toward a greater fraction of massive stars at VHRs because of less efficient cooling due to the lower metallicity of the universe at these early times. 

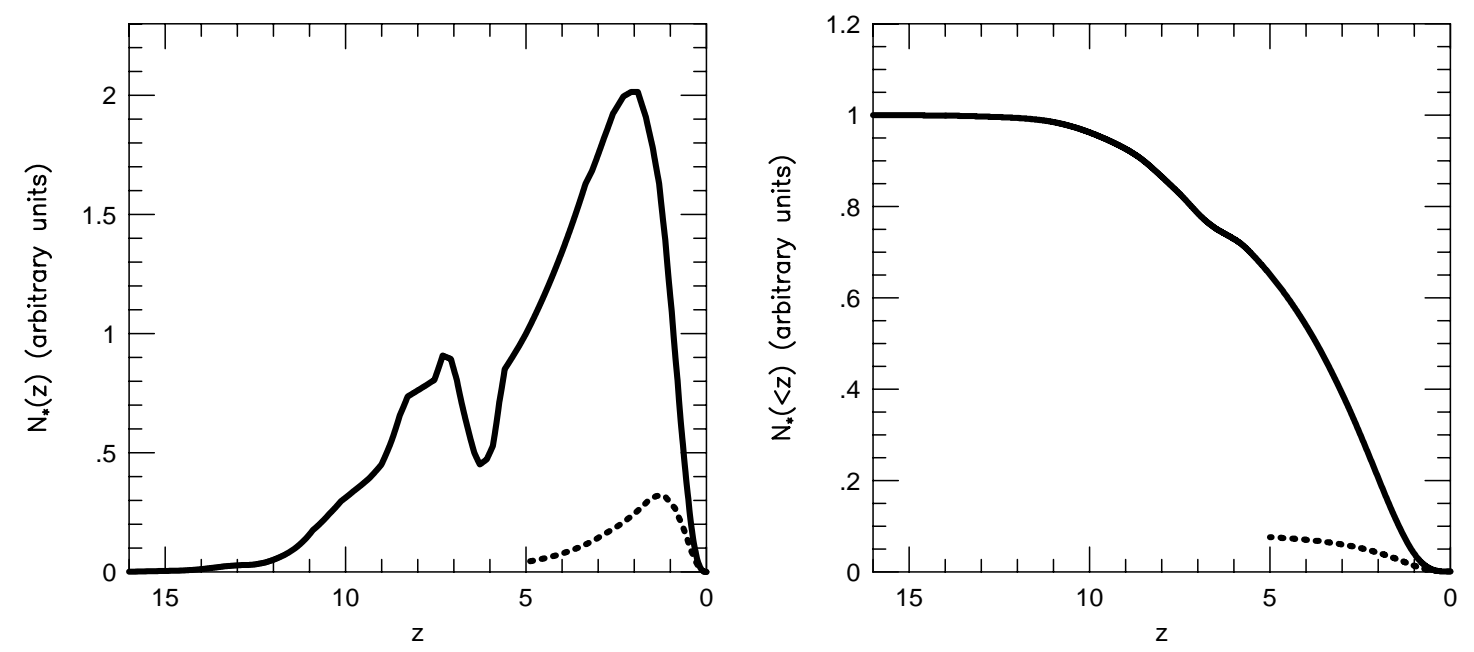

FIGURE 2. Top panel: The number $N_{*}$ of stars expected as a function of redshift $z$ (i.e., the SFR from Figure 1, weighted by the differential comoving volume, and time-dilated) assuming that $\Omega_{M}=0.3$ and $\Omega_{\Lambda}=0.7$. Bottom panel: The cumulative distribution of the number $N_{*}$ of stars expected as a function of redshift $z$. Note that $\approx 40 \%$ of all stars have redshifts $z>5$. The solid and dashed curves in both panels have the same meanings as in Figure 1.

$R_{S F}(z)$ is the local co-moving SFR at $z$, and $d V(z) / d z$ is differential comoving volume [24].

The left panel of Figure 2 shows the number $N_{*}(z)$ of stars expected as a function of redshift $z$ (i.e., the SFR, weighted by the co-moving volume, and time-dilated) for an assumed cosmology $\Omega_{M}=0.3$ and $\Omega_{\Lambda}=0.7$ (other cosmologies give similar results). The solid curve corresponds to the star-formation rate in Figure 1. The dashed curve corresponds to the star-formation rate derived by [19]. This figure shows that $N_{*}(z)$ peaks sharply at $z \approx 2$ and then drops off fairly rapidly at higher $z$, with a tail that extends out to $z \approx 12$. The rapid rise in $N_{*}(z)$ out to $z \approx 2$ is due to the rapidly increasing volume of space. The rapid decline beyond $z \approx 2$ is due almost completely to the "edge" in the spatial distribution produced by the cosmology. In essence, the sharp peak in $N_{*}(z)$ at $z \approx 2$ reflects the fact that the SFR we have taken is fairly broad in $z$, and consequently, the behavior of $N_{*}(z)$ is dominated by the behavior of the co-moving volume $d V(z) / d z$; i.e., the shape of $N_{*}(z)$ is due almost entirely to cosmology. The right panel in Figure 2 shows the cumulative distribution $N_{*}(>z)$ of the number of stars expected as a function of redshift $z$. The solid and dashed curves have the same meaning as in the upper panel. This figure shows that $\approx 40 \%$ of all stars have redshifts $z>5$.

The upper panel of Figures 3 shows the predicted peak photon flux distribution $N_{G R B}(P)$. The solid curve assumes that all bursts have a peak (isotropic) photon luminosity $L_{P}=10^{58} \mathrm{ph} \mathrm{s}^{-1}$. However, there is now overwhelming evidence that GRBs are not "standard candles." Consequently, we also show in Figure 3, as an illustrative example, the convolution of this same SFR and a logarithmically flat photon luminosity function $f\left(L_{P}\right)$ centered on $L_{P}=10^{58} \mathrm{ph} \mathrm{s}^{-1}$, and having widths 

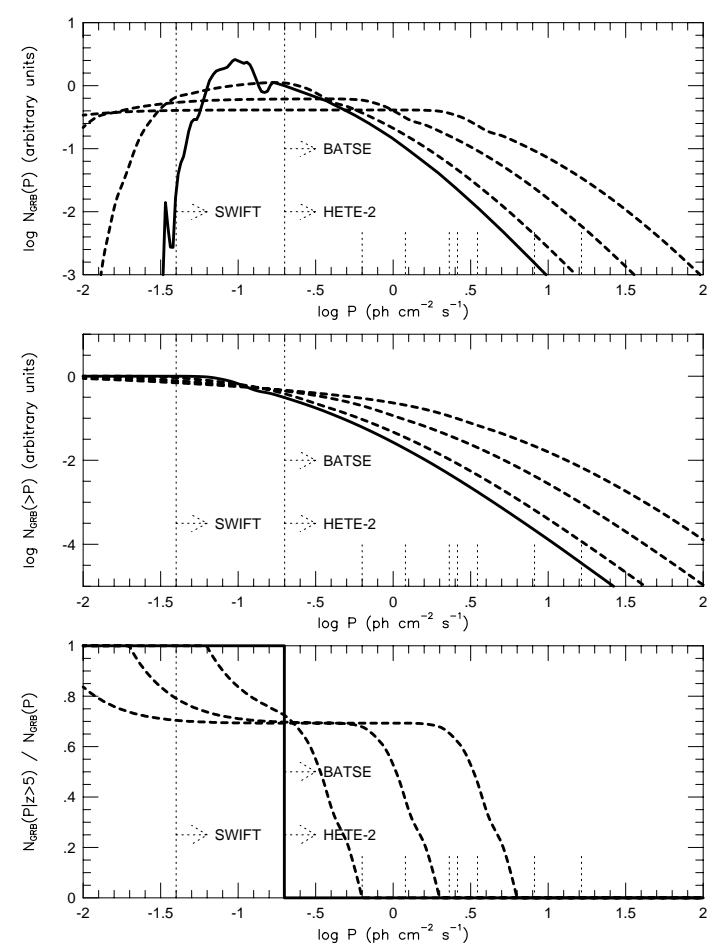

FIGURE 3. Top panel: The differential peak photon flux distribution of GRBs, assuming that (1) the GRB rate is proportional to the SFR, (2) the SFR is that shown in Figure 1; and (3) the bursts are standard candles with a peak photon luminosity $L_{P}=10^{58}$ ph $\mathrm{cm}^{-2} \mathrm{~s}^{-1}$ (solid curve), or have a logarithmically flat peak photon luminosity function that spans a factor of 10, 100, or 1000 (dashed curves). Approximate detection thresholds are plotted for BATSE and HETE-2, and for Swift (dotted lines). Middle panel: The cumulative peak photon flux distribution of GRBs for the same luminosity functions. Lower panel: The fraction of GRBs with peak photon flux $P$ that have redshifts of $z \gtrsim 5$ for the same luminosity functions. In all three panels, the dotted hashes mark the peak photon fluxes of the bursts with known peak photon luminoisities and redshifts .

$\Delta L_{P} / L_{P}=10,100$ and $1000 .^{2}$ The actual luminosity function of GRBs could well be even wider [25].

The middle panel of Figure 3 shows the predicted cumulative peak photon flux distribution $N_{G R B}(>P)$ for the same luminosity function. For the SFR that we have assumed, we find that, if GRBs are assumed to be "standard candles," the predicted peak photon flux distribution falls steeply throughout the BATSE and HETE-2 regime, and therefore fails to match the observed distribution, in agreement with earlier work. In fact, we find that a photon luminosity function spanning at least a factor of 100 is required in order to obtain semi-quantitative agreement with the principle features of the observed distribution; i.e., a roll-over at a peak

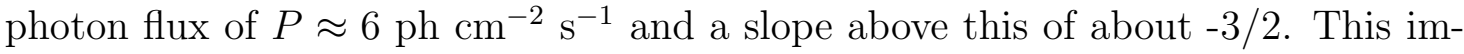
plies that there are large numbers of GRBs with peak photon number fluxes below the detection threshold of BATSE and HETE-2, and even of Swift.

The lower panel of Figure 3 shows the predicted fraction of bursts with peak photon number flux $P$ that have redshifts of $z>5$, for the same luminosity functions. This panel shows that a significant fraction of the bursts near the Swift detection threshold will have redshifts of $z>5$.

2) The seven bursts with well-determined redshifts and published peak (isotropic) photon luminosities have a mean peak photon luminosity and sample variance $\log L_{P}=58.1 \pm 0.7$. 


\section{CONCLUSIONS}

We have shown that, if many GRBs are indeed produced by the collapse of massive stars, one expects GRBs to occur out to at least $z \approx 10$ and possibly $z \approx 15-20$, redshifts that are far larger than those expected for the most distant quasars. GRBs therefore give us information about the star-formation history of the universe, including the earliest generations of stars. The absorption-line systems and the Ly $\alpha$ forest visible in the spectra of GRB afterglows can be used to trace the evolution of metallicity in the universe, and to probe the large-scale structure of the universe at very high redshifts. Finally, measurement of the Ly $\alpha$ break in the spectra of GRB afterglows can be used to constrain, or possibly measure, the epoch at which re-ionization of the universe occurred, using the Gunn-Peterson test. Thus GRBs and their afterglows may be a powerful probe of the very high redshift $(z \gtrsim 5)$ universe.

\section{REFERENCES}

1. Castander, F. J., \& Lamb, D. Q. 1999, ApJ, 523, 593

2. Fruchter, A. S., et al. 1999, ApJ, 516, 683

3. Kulkarni, S. R., et al. 1998, Nature, 395, 663

4. Fruchter, A. S. 1999, ApJ, 516, 683

5. Sahu, K. C., et al. 1997, Nature, 387, 476

6. Kulkarni, S. R., et al. 1999, Nature, 398, 389

7. Reichart, D. E., 1999, ApJ, 521, L111

8. Galama, T. J., et al. 2000, ApJ, submitted (astro-ph/9907264)

9. Bloom, J. S., et al. 1999, Nature, 401, 453

10. Woosley, S. E. 1993, ApJ, 405, 273

11. Woosley, S. E. 1996, in Gamma-Ray Bursts, eds. C. A. Meegan, R. D. Preece, \& T. M. Koshut (New York: AIP), 520

12. Paczyński, B. 1998, ApJ, 494, L45

13. MacFadyen, A. I., \& Woosley, S. E. 1999, ApJ, 524, 262

14. Wheeler, J. C., et al. 1999, ApJ, submitted (astro-ph/9909293)

15. MacFadyen, A. I., Woosley, S. E., \& Heger A. 1999, ApJ, submitted (astro$\mathrm{ph} / 9910034)$

16. Gallego, J. 1995, ApJ, 455, L1

17. Lilly, S. J., et al. 1996, ApJ, 460, L1

18. Connolly, A. J. 1997, ApJ, 486, L11

19. Madau, P., Pozzetti, L., \& Dickinson, M. 1998, ApJ, 498, 106

20. Ostriker, J. P., \& Gnedin, N. Y. 1996, ApJ, 472, L63

21. Gnedin, N. Y., \& Ostriker, J. P. 1997, ApJ, 486, 581

22. Valageas, P., \& Silk, J. 1999, A\&A, 347, 1

23. Rowan-Robinson, M. 1999, Astroph. \& Space Sci., in press (astro-ph/9906308)

24. Lamb, D. Q., \& Reichart, D. E. 2000, ApJ, in press (astro-ph/9912368)

25. Loredo, T. J., \& Wasserman, I. M. 1998, ApJ, 502, 108 